\title{
Noreau, P. et Bernheim, E. (dir.). (2019). Devenir professeur. Montréal, QC : Presses de l'Université de Montréal.
}

D'entrée de jeu, professeurs et doctorants pourraient soutenir que "Devenir prof, ça ne s'apprend pas dans les livres...» (Noreau et Bernheim, 2019, p. 1). C'est néanmoins le sujet traité dans l'ouvrage «Devenir professeur» publié sous la direction de Pierre Noreau et Emmanuelle Bernheim.

Que peut-on attendre d'un tel ouvrage? Certainement qu'il traite des quatre grandes composantes de la vie professorale que sont l'enseignement, la recherche, la vie universitaire et les services à la collectivité. Pourtant, bien que le propos traite implicitement de ces composantes, l'ouvrage, de quelque 400 pages, se divise plutôt en quatre catégories issues des préoccupations abordées par les nombreux auteurs. En effet, on y retrouve une cinquantaine de collaborateurs, provenant de plus d'une dizaine d'institutions d'enseignement supérieur, à l'origine de 35 chapitres, dans un style accessible et direct. Les textes appuyés tantôt par la recherche, tantôt par l'expérience, y sont présentés sous une forme synthétique et vulgarisée.

Le premier tiers de l'ouvrage de Noreau et Bernhiem (2019) est consacré à la première des quatre catégories ayant émergé des contributions, celle sur l'enseignement. Les collaborateurs y abordent la planification de l'enseignement, incluant les contenus à enseigner et l'élaboration du plan de cours, les stratégies d'enseignement et celles d'évaluation des apprentissages. Par la suite, l'aspect relationnel du climat de classe et la capacité à s'adapter en fonction des conditions d'enseignement, comme la taille des groupes, sont mis de l'avant. Trois chapitres sont ensuite consacrés aux particularités de l'enseignement et de l'encadrement individuel et collectif des étudiants aux cycles supérieurs, alors que deux autres attirent l'attention sur la place des technologies de la communication dans l'enseignement. Cette section 
se conclut en présentant l'évaluation de l'enseignement et l'innovation comme des outils d'amélioration de l'enseignement.

La recherche et son rayonnement regroupent les textes de la deuxième section. Cette partie s'emploie à expliquer « pourquoi » et " comment » réaliser les activités liées à la recherche, ainsi que la place des étudiants de cycles supérieurs dans ce processus. Au fil des chapitres, l'attention du lecteur se porte sur la question des droits d'auteur, sur l'importance de la communication scientifique et, surtout, sur les différents aspects du processus de publication. Les auteurs parlent notamment de la publication en libre accès, ainsi que des particularités de la publication en contexte scientifique, professionnel et grand public, avant de terminer en abordant la complexité de la rédaction des demandes de subvention.

La troisième section concerne la carrière professorale, entre autres, du point de vue de la multiplication des rôles. Dans l'ordre, les chapitres décrivent des situations vécues par les professeurs comme : être prof et doctorant, prof et à la direction d'un département ou d'un programme, prof et impliqué auprès du syndicat, prof et à la direction d'une revue ou, simplement, prof et collègue. S'ensuivent des textes sur la fragmentation de la tâche professorale, ce qui découle sur des enjeux de conciliation travail-famillevie personnelle, sur le dilemme d'accepter ou de refuser les multiples opportunités qui se présentent, sur la présence physique à l'université et sur la question de la formation pour enseigner des professeurs universitaires.

La quatrième et dernière section de l'ouvrage «Devenir professeur » comporte des textes à propos des défis et des figures imposées, autrement dit, sur les pièges à éviter dans la carrière en milieu universitaire. Les sujets explorés vont de la prise en compte de la diversité à la sensibilité aux problèmes de santé mentale des étudiants, en passant par les défis liés à l'intégration des professeurs d'origine étrangère. Il y est également question du rapport à l'écrit du professeur, des balises de la liberté académique, de la prise de parole dans le débat public et des risques de conflits d'intérêts associés aux fonctions professorales.

Ce livre se destine résolument aux aspirants et aux plus ou moins récents membres du corps professoral universitaire, et ce, de toutes les disciplines. A posteriori, louvrage fournit, aux membres et futurs membres du corps professoral, un portrait global de ce qui compose la tâche du professeur universitaire. S'il faut reconnaitre qu'un chapitre d'une dizaine de pages ne suffit pas à expliquer en profondeur l'une ou l'autre des thématiques abordées, cette formule a le mérite de mettre en évidence la complexité du rôle endossé. Pour le résumer simplement, l'ouvrage brosse large, mais il ne permet pas de cibler les priorités parmi les nombreux enjeux abordés. En plaçant plus ou moins tout au même niveau d'importance, le lecteur pourrait bien craindre d'y laisser sa peau à l'idée de « Devenir professeur »!

Cette idée nous ramène aux limites d'un ouvrage pour décrire et comprendre une profession, ainsi qu'à la question des destinataires. Et si les plus chevronnés des professeurs retrouvaient dans cet ouvrage, comme un rappel, les préoccupations de leurs collègues novices? Voilà qui placerait ce livre entre de multiples mains, comme guide pour les aspirants et nouveaux professeurs, mais également comme matière à nourrir l'importance du rôle de mentor pour accompagner les professeurs en début de carrière.

\section{Pour citer cet article}

Gagné, A. (2020). Noreau, P. et Bernheim, E. (dir.). (2019). Devenir professeur. Montréal, QC : Presses de l'Université de Montréal. Formation et profession, 28(3), 137-138. http://dx.doi.org/10.18162/fp.2020.a218 